\title{
Présentation
}

Presentation

\section{Patrice Duran}

\section{(2) OpenEdition}

Journals

Édition électronique

URL : https://journals.openedition.org/ress/4876

DOI : $10.4000 /$ ress. 4876

ISBN : 1663-4446

ISSN : 1663-4446

Éditeur

Librairie Droz

Édition imprimée

Date de publication : 30 mai 2019

Pagination : 13-16

ISSN : 0048-8046

\section{Référence électronique}

Patrice Duran, «Présentation », Revue européenne des sciences sociales [En ligne], 57-1 | 2019, mis en ligne le 30 mai 2019, consulté le 04 janvier 2023. URL : http://journals.openedition.org/ress/4876 ; DOI : https://doi.org/10.4000/ress.4876 


\section{PRÉSENTATION}

\section{PAR PATRICE DURAN}

CNRS - ENS Paris Saclay, Institut des sciences sociales du politique

La portée de l'œuvre de Max Weber est immense qui diffuse dans l'ensemble des sciences sociales. Lui-même a su croiser histoire, droit, économie et sociologie, qu'il définit comme des sciences de la réalité (Wirklichkeitwissenschaften) afin d'en marquer le socle empirique commun. Cherchant à combiner la mise au jour des régularités sociales en même temps que l'explication de la singularité des processus historiques, Weber a développé des orientations de recherche très puissantes qui se situent encore aujourd'hui au cœur de la réflexion des diverses disciplines des sciences sociales dont il a su ainsi montrer la porosité des frontières et l'utilité de la confrontation.

Aujourd'hui, non seulement la place occupée par Weber dans les sciences sociales est restée très actuelle, mais la connaissance de son œuvre s'est fortement enrichie au cours des vingt dernières années. On sait à quel point il est difficile de dissocier, pour Weber plus que pour d'autres, le travail d'interprétation de l'œuvre de son histoire éditoriale. Longtemps, ses écrits volumineux et très dispersés n'étaient accessibles que dans des éditions partielles et souvent peu fiables. Du même coup, l'exercice de reconstruction et d'interprétation n'était jamais aisé ni assuré. C'est grâce au travail éditorial exemplaire réalisé en Allemagne sur l'œuvre de Weber dans le cadre de la Max Weber-Gesamtausgabe (MWG) que la question des textes de Weber, de leur nature, de leur contexte, de leur chronologie a bénéficié de nouveaux éclairages. Il n'est par conséquent guère surprenant que cela ait considérablement stimulé la recherche sur l'œuvre de Weber comme en témoignent tout particulièrement le Max Weber Handbuch sous la direction de Hans-Peter Müller et Steffen Sigmund (2014) et la sortie prochaine du Oxford Handbook of Max Weber édité par Edith Hanke, Lawrence A. Scaff et Sam Whimster. De même, de nouvelles traductions françaises ainsi que des retraductions de certains textes ont ainsi été réalisées à partir des éditions critiques réalisées en Allemagne. 
La contribution de François Chazel vise justement, grâce au travail effectué dans le cadre de la MWG, à dresser un bilan des textes essentiels de Weber consacrés à la sociologie de la domination et saisir l'apport spécifique des deux versions de la sociologie de la domination qui se dégagent de la lecture de Wirtschaft und Gessellschaft. Question centrale que celle de la domination dès lors que, comme l'écrit Weber lui-même, la domination est «le fondement le plus important de presque toute activité de groupement» (Weber, I982, p.470). Ainsi que Chazel le souligne, il ne s'agit pas de rejeter l'une pour préférer l'autre. Chacune reflète les intérêts de Weber. Il est vain de chercher une complémentarité des deux ensembles, on y voit plutôt la variation des intérêts de connaissance de Weber lui-même en fonction de ses propres interrogations à des moments historiques spécifiques tout à la fois de son œuvre et de l'histoire dans laquelle il vit. Si la première est tournée vers une sociologie d’ensemble du développement vers la modernité, la seconde est davantage une clarification conceptuelle ordonnée autour du noyau central des types de domination légitime.

Patrice Duran revient, en ce qui le concerne, sur la question de la légitimité politique, centrale dans la seconde version de la sociologie de la domination. L’absence de définition du concept lui-même montre que la typologie élaborée n'est qu’une des dimensions de la légitimité qui s’apparente en fait à un processus complexe dont Weber précisera la portée dans une sociologie de l'État qui n'est pas étrangère à l'expérience qu'il aura de la difficile construction de l'État national allemand. Weber a su marquer de manière décisive la faiblesse et la fragilité de la légitimité dès lors qu'il n’existe pas de fondement définitif de l'ordre politique dans un monde sécularisé. Il a ouvert ainsi une réflexion sur la question cruciale de l’acquisition de la légitimité qui s’avère bien utile pour comprendre le déficit ou simplement les variations de légitimité qui affectent les modes de gouvernement actuels.

Quant à Andreas Anter, il atteste clairement l'influence qui a pu être celle de la pensée politique de Weber sur la Constitution même de la République fédérale d’Allemagne. Non seulement on a pu y trouver une défense puissante de la démocratie représentative qui conduit à remiser une interprétation le plus souvent caricaturale d'une démocratie s'appuyant sur des chefs plébiscitaires, mais on y trouve 
également la preuve d'un réalisme politique directement inspiré d'un réalisme sociologique sans faille dans la défense d'un fédéralisme unitaire susceptible d'assurer l'existence d'un État garant d'un ordre politique qui assure l'unité nationale en même temps que la protection de la diversité de ses composantes.

Hinnerk Bruhns de son côté montre bien la place que tient l'histoire dans la réflexion wébérienne; une histoire qu'il faut comprendre et intégrer dans la pensée de l'avenir. La comparaison avec Shmuel N. Eisenstadt du point de vue de l'élaboration des programmes de recherche sur la modernité est à cet égard parfaitement éclairante sur les rapports entre théorie sociologique et histoire chez Weber. On le voit dans le refus de Weber d'accorder à la religion le rôle de facteur décisif dans le développement de la modernité. De même, le réalisme sociologique wébérien conduit ce dernier à accepter implicitement la pluralité des modernités et à ne pas confondre toute la modernité avec une modernité qui serait celle de l'occident, laquelle ne peut se comprendre à partir d'un angle seulement macro-sociologique. Analyser et rendre compte d'un processus de modernisation suppose par conséquent un important travail théorique et méthodologique susceptible de fonder un regard comparatif sur des réalités diverses à même de déboucher sur des modalités de développement différentes.

C’est d'ailleurs la force de la sociologie que de n'être pas vouée à une quelconque spécialisation de son regard. Ainsi sur une question qui est a priori plus celle de l'économiste que du sociologue, la monnaie, Michel Lallement trouve dans le travail de Weber l'expression manifeste d'une sociologie économique dont l'apport s'avère déterminant à l'explication des faits économiques sans négliger pour autant l'apport d’autres sciences sociales. Même si la place assignée à la monnaie reste marginale dans son œuvre, Weber a su fournir un éclairage original sur la nature, les fonctions et les usages sociaux de la monnaie que seul lui permet la pratique d'une sociologie ouverte empruntant autant en économie à l'école marginaliste autrichienne qu'à l'école historique allemande, voire même plus ponctuellement au marxisme pour expliquer aussi bien l'apparition des crises économiques que des phénomènes sociaux aussi complexes que l'inflation ou la spéculation. L'économie ne peut être enfermée dans la seule rationalité en finalité et doit rester ouverte à d'autres exigences 
tout comme on ne saurait l'enfermer dans une seule logique de rationalisation formelle qui ignorerait la rationalisation matérielle à l'œuvre dans toutes les sociétés. Au fond, pourrait-on dire, Weber trouve dans sa sociologie une manière de continuer l'analyse de l'économie par d'autres moyens!

Enfin, dans une très courte note, François Chazel revient sur ce qui était jusque-là une énigme, à savoir la paternité de la formule célèbre de la fin de l'Éthique protestante et l'esprit du capitalisme, «Spécialistes sans esprit, jouisseurs sans cœur: ce néant s'imagine s'être élevé à un degré de l'humanité encore jamais atteint». Après avoir longtemps cru qu'elle était empruntée à Nietzsche et l'avoir cherchée en vain dans toute son ouvre, il a bien fallu admettre que la citation ne pouvait lui être imputée. Or, dans un travail récent, un professeur d'histoire moderne et contemporaine, Hans-Kristof Kraus, indique avoir trouvé dans celle de Gustav Schmoller une formulation très proche. Restituée de mémoire par Weber, la citation s'en est trouvée modifiée d'une manière qui illustre clairement à quel point la pensée de ce dernier restait hantée par un futur fondamentalement incertain.

La portée d'un «classique» se juge à la fécondité de ses approches et de ses programmes de recherche, tout comme à l'aune des perspectives d'analyse qu'il continue d'ouvrir. L'intérêt pour de tels classiques participe moins d'un quelconque devoir de mémoire que de la volonté de les questionner au regard de l'actualité des enjeux théoriques dont ils sont les porteurs et de la force des analyses qu'ils ont proposées. C'est bien ce qui a guidé les auteurs dans la construction de ce dossier. Assurément Max Weber est un «classique», et on voit bien à quel point il est encore possible d'aller avec Weber au-delà de Weber !

\section{BIBLIOGRAPHIE}

HANK E., STAFF L. A. et WHIMSTER S. (dir.), Oxford Handbook of Max Weber, Oxford, Oxford University Press, à paraittre.

MÜLLER H.-P. et SIGMUND S. (dir.), 2014, Max Weber Handbuch, Leben - Werk Wirkung, Stuttgart-Weimar, Metzler.

WEBER M., 1982 (1903-1919), Gesammelte Aufsätze zur Wissenschaftslehre, Tübingen, J.C.B. Mohr (Paul Siebeck). 